\title{
Multimodalität im sozio-kulturellen Kontext: mit Bildern reden, Bilder verstehen
}

\section{Multimodality in Socio-cultural Contexts: To Talk with Pictures, to Understand Pictures}

\author{
Valeria CHERNYAVSKAYA ${ }^{1}$ [D, Ludmila V. KULIKOVA ${ }^{2}$ [D
}

'Prof. Dr., Peter the Great St. Petersburg Politechnic University, Institute of Humanities, St.Petersburg, Russia ${ }^{2}$ Prof. Dr., Siberian Federal University, Institute of Intercultural Communication, Krasnojarsk, Russia

ORCID: V.C. 0000-0002-6039-6305;

L.V.K. 0000-0002-1622-8304

\section{Corresponding author:}

Valeria CHERNYAVSKAYA,

Peter the Great St. Petersburg Politechnic

University, Institute of Humanities,

St. Petersburg, Russia

E-mail: tcherniavskaia@rambler.ru

Submitted: 08.04.2020

Accepted: 13.05 .2020

Citation: Chernyavskaya, V. \& Kulikova, L. V. (2020). Multimodalität im sozio-kulturellen Kontext: mit Bildern reden, Bilder verstehen. Alman Dili ve Edebiyatı Dergisi Studien zur deutschen Sprache und Literatur, 43, 19-38.

https://doi.org/10.26650/sdsl2020-0009

\section{ABSTRACT (DEUTSCH)}

Der vorliegende Beitrag will zur Diskussion über die Multimodalität beitragen mit dem Ziel, auf die visuelle Ressource als notwendige Voraussetzung für das Verstehen hinzuweisen. Er fokussiert die Fragen, wie ein Bild im Diskurs wirksam wird, wie Kommunikationsteilnehmer in einem bestimmten Kulturraum Bedeutungen mithilfe einer visuellen Ressource ausdrücken und interpretieren, welche gesellschaftlich sinnvollen Bedeutungen mit dem im Text enthaltenen Bild verbunden sind. Die Auseinandersetzung mit dem Visuellen bietet einen ,Access Point' zur Kontextualisierung und zugleich eine Evidenzbasis in der theoretischen Begründung dessen, wie das gemeinsame, in der kulturellen Praxis geteilte Wissen entsteht und weiter funktioniert. Die methodologischen Grundlagen werden durch die soziale Semiotik von M. A. K. Halliday, G. Kress, van Leeuwen festgelegt, beruhen auf den theoretischen Vorstellungen von Multimodalität von $\mathrm{H}$. Stöckl, H. Schmiz, B. Sandig, folgen der diskurssensitiven kulturwissenschaftlichen Linguistik nach $\mathrm{H}$. Kuße. Fallbeispiele aus der deutschen und russischen kommunikativen Praxis werden analysiert. Die Expansion des Bildes in sozialen Praktiken erfordert von den Teilnehmern der Kommunikation reflektierte visuelle Erfahrung und eine multimodale Kompetenz. Eine solche Kompetenz beinhaltet die Fähigkeit, ideologische, politische, kulturspezifische Implikationen von Bildern abzuleiten und zu interpretieren, die Werteinstellungen zu verstehen, die hinter der visuellen Gestaltung der Bedeutungen stehen.

Schlüsselwörter: Multimodalität, Visualität, soziale Semiotik, Kultur, sozialer Kontext

\section{ABSTRACT (ENGLISH)}

The present paper is inspired by the challenges posed by multimodal/multi-semiotic resources in the investigation of communication. We focus on the visual resource as a socially and culturally shaped semiotic resource of making meaning and discuss how images work in different socio-cultural contexts. The interpretative complexity of the notions of 'culture' and 'socio-cultural context' is explained by looking at images as they represent information from a socio-cultural environment. The 
theoretical framework of the paper was rooted in Halliday, Kress and Leeuwen's theory of social semiotics, in the concepts of multimodal communication and text linguistic view of the language-image-link according to Stöckl, Schmiz, Sandig, and in Kuße's discourse sensitive cultural linguistics. The questions under discussion are how we communicate through visual images, and how communicative actors produce and interpret meanings through pictures. We use the multimodal texts from modern German and Russian discourse and discuss the role of the visual image in shaping our socio-cultural experience and knowledge. The paper argues for more sensitivity in respect of the expansion of visual images with regard to multimodal competence. The latter plays a crucial role in exploring the ideological and culturally specific implicatures behind the visual images.

Keywords: Multimodality, visuality, social semiotics, culture, social context

\section{EXTENDED ABSTRACT}

In the present paper, we will engage with the important challenges posed by multimodal/multi-semiotic resources in communication. Multimodality is considered as a cultural technique and a communicative competence that guarantees mutual transparence and explicitness for interactors. We focus on the visual resource as a socially and culturally shaped semiotic resource of making meaning. In this framework the paper addresses the way knowledge and values are constructed through images as well as language usages.

The paper attempts to point out a socio-cultural perspective on the understanding of multimodal texts. The paper discusses how images, as part of language-image-links, work in different socio-cultural contexts and shape our sociocultural experience and knowledge. Thus, the goal is to look at images as they represent information from a sociocultural environment. The interpretative complexity of the notions of 'culture' and 'sociocultural context' is explained. The analysis follows the position of discourse oriented cultural linguistics that presumes the situational and socio-cultural framework both for the production and the reception of utterances/texts.

Methodology. Several approaches to multimodal communication have been developed. The theoretical framework of this paper was found in the theory of social semiotics according to Halliday, Kress, van Leeuwen, and in the concepts of multimodal communication and text linguistic view of the language-image-links according to Stöckl, Schmiz, Sandig and others. The present analysis is also in line with Kuße's discourse sensitive cultural linguistics (diskurssensitive kulturwissenschaftliche Linguistik). From this theoretical perspective we show that there is a shift from the "linguist's pride in language" (in Stöckl's words) towards multimodality and visual methodology in meaning 
construction. We follow social semiotics in looking at how verbal and nonverbal signs (semiotics resources) are used in certain social practices. It has been emphasized in social semiotics how semiotic practices are driven by socio-cultural environments and how they are embedded in them and emerge from them. The paper looks at multimodal texts and images as they are context sensitive.

Findings and Results. The theoretical reflections and text analytical observations show the explanatory charge of multimodal studies. In the provided analysis we tackle the multimodal texts and cases in point from modern German and Russian socio-cultural practice. Different types of image-language-links are considered, following the basic observations in text linguistics, namely complement and discrepancy or contrast. Multimodal metaphors and metonymy are also discussed. Visual images are iconic and also indexical (i.e. metonymic) in terms of their representation of abstract concepts and objects/events in their socio-cultural embeddedness.

The authors presume that pictures should be understood in context and in combination with language. Linguistic and visual resources cooperate as they interrelate concepts. From this point of view, images are seen as an additional access point to explain meaning making process within its cognitive dimension. Next, multimodal texts, and images as a part of these texts, allow us to observe how socio-cultural work is performed. This, in turn, leads to the conclusion, that images are context embedded. In other words, they present information from the social environment. A visual image is not just an information vehicle. It is a constituent element of dynamic sense-making process. The cases under discussion show that multimodal texts could act as wrong indexicals and may cause wrong contextualization, if they are misplaced in contexts, i.e. if the context of text reception does not fit the context of text production. We can make sense of imagelanguage-texts if they fit the context.

The present paper argues for more sensitivity in respect of the expansion of visual images and with regard to multimodal competence. The multimodal competence plays a crucial role in exploring the ideological and culturally specific implicatures behind the visual images. A potentially wide perspective integrating verbal text and picture within different social practices should also be considered as helpful in this framework. 


\section{Einleitung}

Der Gegenstand des Beitrags ist Multimodalität, wobei wir uns auf die sprachliche und visuelle Modalität beschränken, d.h. auf Wort-Bild Kommunikation. Der theoretische Kontext der Analyse wird durch das moderne Verständnis von der multimodalen Natur der menschlichen Kommunikation bestimmt. Im humanitären und sozialen Wissen wurde die methodische Priorität von der Anerkennung der Sprache als Hauptkommunikationscode auf die Untersuchung anderer Zeichenmodalitäten umgesetzt, und zwar einer visuellen Präsentation von Bedeutungen und Identitäten. Visualität bedeutet die semiotische Ressource zur Sinngebung, die sich im Rahmen einer bestimmten Kultur entwickelt und sozial geprägt hat, vgl.: « socially shaped and culturally given semiotic resource of making meaning » (Kress, 2010, S. 79). Dieser Trend ist auch für viele Ansätze in der Linguistik klar. Noch vor einem Jahrzehnt stellte H. Stöckl fest, die Linguistik stehe noch unter Druck, die multimodale Wende zu legitimisieren, vgl.: "linguistics is still under quite some pressure to legitimize the pictorial or multi-semiotic turn" (Stöckl, 2009, S. 203). Jetzt ist die Forschungssituation anders. Der mulimodale Ansatz liefert starke Impulse, um die dynamische Bedeutungskonstruktion detailliert zu erforschen, wobei das Visuelle mitwirkt.

In den Geistes- und Sozialwissenschaften lässt sich heute die wachsende Beliebtheit visueller Analysetechniken feststellen, die sich in einer Vielzahl wissenschaftlicher Fachzeitschriften zeigt, z.B.,Visual Communication',,Visual Studies','Visual Anthropology', ,Visual Anthropology Review',,The Journal of Visual Culture' etc. Experten sprechen von "visueller Kommunikation", „visueller Argumentation", "visueller Rhetorik", "visueller Metapher", "visuellem Diskurs" als neuen Objekten der Analyse, einschließlich neuer Zugangspunkte zum Verständnis von dynamischen Prozessen der Sprachkommunikation. Die Bedeutung der visuellen Darstellung in der angewandten Forschung hat durch die Entwicklung neuer medialer Formate, visueller Technologien und elektronischer Medien, sogenannter digitaler Kultur, zugenommen. Dadurch wird auch die akademische Diskussion in Semiotik, Linguistik, Kommunikationstheorie, Anthropologie und Kulturwissenschaft angeregt. Zusammen mit der Weiterentwicklung der Diskursanalyse hat die Multimodalität als Schlüsselbegriff für die Erklärung der Prozesse des Verstehens und der Bedeutungsbildung das Monopol der Verbalität, des Logozentrismus abgelöst (Kress, 2010; Kress \& van Leeuwen, 1996 (2006); 2001; Stöckl, 2009; Schmiz, 2004). 
In der heutigen Informationsgesellschaft basiert die Realität auf unserem Wissen darüber. Die Assoziationen, Erfahrungen, also der Diskurs, sind notwendig, weil nur dank diesen es möglich ist, eine einzelne Aussage - das "hier und jetzt“ des Geäußerten - angemessen zu verstehen. Visuelle Information und visuelle Erfahrung der Kommunikationsteilnehmer müssen als wesentlich für die Bedeutungskonstitution angesehen werden.

Das Ziel des Beitrags ist es zu zeigen, wie das visuelle Bild zur Konstruktion menschlicher Erfahrungen und Kenntnisse beiträgt. Die Ausführungen wollen zur Sensibilisierung der Kommunikationsteilnehmer für komplexes Zusammenwirken zwischen Sprache und Bild, einerseits, und zwischen Zeichenmodalitäten in der Kommunikationssituation, d.h. ihrer Kontextualisierung in der sozio-kulturellen Praxis, andererseits. Der vorliegende Beitrag fokussiert die Fragen, wie ein Bild im Diskurs wirksam wird; wie Kommunikationsteilnehmer in einem bestimmten Kulturraum Bedeutungen mithilfe einer visuellen Ressource ausdrücken und interpretieren; welche gesellschaftlich sinnvollen Bedeutungen mit dem im Text enthaltenen Bild verbunden sind.

\section{Methodologie}

Die methodologischen Grundlagen der Analyse werden im weitesten Sinne durch die soziale Semiotik in der Tradition von M. A. K. Halliday, G. Kress, van Leeuwen festgelegt. Die Ausführungen beruhen auf den theoretischen Vorstellungen von Multimodalität von H. Stöckl, H. Schmiz, B. Sandig, folgen der diskurssensitiven kulturwissenschaftlichen Linguistik nach $\mathrm{H}$. Kuße.

Das Thema wird in drei Schnitten eingegangen. Zunächst werden theoretische Grundlagen und Schlüsselbegriffe zur Analyse vorgestellt, daran anschließend die wesentlichen Herangehen zu Sprache-Bild-Verknüpfungen gezeichnet und mit Fallbeispielen aus der deutschen und russischen kommunikativen Praxis analysiert, um abschließend zu zeigen, wie multimodale Texte und Bilder als ihr Bestandteil in soziokulturellen Kontexten funktionieren und an der Sinnbildung beteiligt sind.

\section{Theoretische Grundlagen}

Den Ausgangspunkt für Diskussionen liefert der Begriff,Kultur'.

Mit Kultur bezeichnet man im weiten Sinne alles, was der Mensch selbst gestaltend 
schafft, seine materiellen und geistigen Errungenschaften, d. h. alle Formen der Kognition und Kommunikation in der Gesellschaft. Es sollte anerkannt werden, dass Verweise auf kulturelle Unterschiede und der Begriff "kulturelle Spezifik“ von vielen Autoren generell als nützlich angesehen werden, um die Phänomene zu beschreiben, die wir in der sozialen Praxis, im sozialen Kontext beobachten. Offensichtlich das allererste, was zur Diskussion der Konzepte von Kommunikation und Kultur vorgebracht wird, ist die Hypothese der sprachlichen Relativität von Sapir und Whorf mit ihrer Hauptposition: Sprache beeinflusst die Art und Weise, wie wir die Welt verstehen, und die erste explizite Formulierung des sprachlichen Relativitätsprinzips von Humboldt, demzufolge sprachliche Strukturen das Denken strukturieren. Im Weiteren wurde dies seit den 1980er Jahren in einer "linguistischen Wende" im sozialen und humanitären Wissen ausführlicher formuliert, vgl. ausführlich in (Chernyavskaya, 2014; Kuße, 2012; Kuße \& Chernyavskaya, 2019; Kulikova, 2019).

In den vorliegenden Ausführungen liegt der Forschungsschwerpunkt auf einer anderen Perspektive und einer anderen theoretischen Strategie zum Verständnis kultureller Besonderheiten. Die Hauptthese hier lautet: Unser Verständnis basiert nicht nur auf der Bedeutung sprachlicher Einheiten und der Grammatik der Sprache, sondern auch auf außersprachlichem Wissen über die Welt, auf sozialen und kulturellen Voraussetzungen, die zusammenwirken. Den Worten von $\mathrm{H}$. Kuße folgend , Kultur ist eine Form von Erkennen durch Bedeutungszuschreibung. Aus der Notwendigkeit der Bedeutungszuschreibung und der Zeichenhaftigkeit aller kulturellen Gegenständen folgt der enge Zusammenhang von Kultur und Kommunikation. ... Ohne Kommunikation über Artefakte kann es keine kulturelle Bedeutungszuschreibung geben" (Kuße, 2012, S. 29, 35-36). Wie Spitzmüller betont, ,Kultur' kann also als diskursiv produziertes Orientierungssystem verstanden werden, das es ermöglicht, Handlungen und Handlungsresultate zu bewerten und im sozialen Raum (horizontal und vertikal) zu positionieren - und mithin auch die eigenen Handlungen und sich selbst als Handelnden sozial einzuordnen. Kultur ist also das Produkt, aber auch der Kontext von Diskursen (Spitzmüller, 2017, S. 13).

Um die Kultur zu verstehen, ist der Schwerpunk wichtig, der erstens auf der kognitiven Projektion der Analyse liegt; zweitens auf der sozialen Projektion, da Kultur als Regelwerk gesellschaftsbezogen wirkt; und drittens auf semiotischen Ressourcen für den Ausdruck und die Gestaltung von kulturell bedeutenden Phänomenen. Es ist von grundlegender Bedeutung, dass Kultur nicht als statisches Phänomen, sondern als diskursives Konstrukt 
analysiert wird: es ist ein Prozess der sozialen Konstruktion, in dem die Identität, die Werte und Normen geformt und zur Geltung gemacht werden. Mehr darüber in (Kuße \& Chernyavskaya, 2019; Molodychenko, 2015; 2017; Widdowson, 2004).

Die Frage, wie das visuelle Bild in der kulturspezifischen kommunikativen Praxis funktioniert, wurde zentral und in jenen Studien bereits weitgehend reflektiert, die sich seit Ende der 1980er Jahre im Rahmen der sogenannten visuellen (ikonischen) und multimodalen Wende (vgl.: visual turn, iconic turn, pictorial turn, multimodal turn) in den Sozial- und Geisteswissenschaften entwickelt haben. Der Begriff "Wende“ bezeichnet dabei eine Verschiebung des Fokus auf einen anderen Gegenstand. Gleichzeitig bedeutet das eine kritische Auseinandersetzung mit einem neuen Thema.

Die visuelle Wende ist, wie die Experten meinen, ein Gegengewicht zur sprachlichen Wende. Die linguistische Wende oder Wende zur Sprache hat ihrerseits die grundlegende Rolle der Sprache bei der Konstruktion unserer Vorstellungen von der Realität in den Vordergrund gestellt und postuliert, dass die Erkenntnis durch die Sprache strukturiert wird. Der visuelle Trend der Forschung hat zu der These geführt, dass wir einen direkten unmittelbaren Kontakt mit der Welt brauchen, der nicht durch unsere Sprache vermittelt wird. Der Schwerpunkt hat sich von der Anerkennung der Sprache als Hauptcode und dem ,Archimedium der Kommunikation' nach K. Ehlich (Ehlich, 1998, S. 20) auf die Untersuchung anderer Zeichenmodalitäten umgesetzt, und zwar auf die visuelle Repräsentation von Bedeutungen und Identitäten.

Medialität ist die Bindung der Bedeutung an ein bestimmtes Format, ihre Ausgestaltung durch besondere semiotische Ressourcen. Hier ist das Verständnis der Medialität nicht nur als Mittel zur Übertragung von Bedeutungen, sondern als Kanal der Sinnbildung grundsätzlich wichtig. Multimodalität ist ihrerseits ein wesentliches Kommunikationsprinzip, das die Kommunikation als solche ermöglicht, d.h. verständlich und erreichbar für ihre Teilnehmer macht, die „Unumgänglichkeit der menschlichen Kommunikation“ zeigt (Stöckl, 2010, S. 47), vgl. auch: «multimodality is a cultural technique, a competence which guarantees communication and mutual intelligibility» (Stöckl, 2009, S. 206).

Das visuelle Format hat seine eigene semiotische Ressource und seine alternative Logik für die Gestaltung von Bedeutungen. Die wichtige Forschungsfrage ist, was bringt der visuelle Modus in die Kommunikation? Von welcher Bedeutung ist die Funktionsteilung zwischen Wort und Bild? 
Bilder sind prototypischerweise ikonisch, Sprachtexte sind mit symbolischen Zeichen gebildet, die sich auf Konventionen beruhen. Das Bild bietet uns direkten Kontakt mit der Welt. Der visuelle Informationskanal reduziert die mögliche Asymmetrie des Zeichens und der vom Zeichen reflektierten Welt, die durch die Qualität des kommunikativen Kanals erzeugte Diskrepanz, vgl. « reduces the discrepancy between the world depicted and the medium used» (Stöckl, 2009, S. 205).

Die Ikonizität des Visuellen unterstützt den sachlichen Charakter, die Zuverlässigkeit der durch visuelle Mittel zum Ausdruck gebrachten Botschaft. Das Bild ähnelt äußerlich der materiellen Realität. Das Visuelle in verschiedenen Formen - Malerei, Fotografie, Video, Dokumentationen usw. ist am Aufbau von Vertrauen, Beweisen beteiligt. Es wird ein besonderer Effekt oder eine besondere Art der Anwesenheit des Rezipienten in der reflektierten Realität erzeugt. Das Bild wirkt als Instrument der Offenheit und Transparenz.

Wenn man die semiotischen Eigenschaften im visuellen Format mit dem sprachlichen vergleicht, sollte man berücksichtigen, wie die begrifflichen (denotativen) und konnotativen Komponenten unterschiedlich ausgedrückt werden. Das Überwiegen der emotional-bewertenden Komponente im Bild macht es bei der Umsetzung von der expressiven, bewertenden Funktion der Aussage flexibel und ermöglicht es, die Situation als lebendig und dynamisch, mit oft unlogischen Beziehungen einzufangen und zu fixieren.

Damit sind wir bei einem wesentlichen Punkt. Das visuelle Bild ist keine Alternative zum logozentrischen Ansatz, es vereinfacht unsere Erfahrung nicht, sondern ist in die komplexe Verbindung der multimodalen Aussage mit ihrem soziokulturellen Hintergrund integriert. Visuelle Zeichen und Formen werden aktiv verwendet, um Werte und Identitäten zu konstruieren und zu repräsentieren.

Wir gehen davon aus, dass das visuelle Bild nicht nur die Realität widerspiegelt, sondern auch bestimmte Beziehungen zwischen dem Adressaten und dem Empfänger herstellt. In einer sozio-semiotischen Perspektive ist das Bild ein Teil der sozialen Praxis. Um das Bild zu verstehen, braucht der Empfänger einen Kontext, in den das Bild als seine Komponente und seine Reflexion integriert ist. Im sozialen Raum wird das Visuelle neben der sprachlichen Bedeutung zu einem wesentlichen Element sozialer Strukturen und zu einem Teil der Sinngebung. 


\section{Analyse und Diskussion}

\subsection{Sprache-Bild Kommunikation: Verknüpfungsmodelle}

Das Bild wurde Gegenstand einer Reihe von Spezialstudien zur pragmatisch und funktional orientierten sprachlichen Analyse, zur Stilistik und zur Textlinguistik. Es wurde vom Standpunkt untersucht, wie es zusammen mit sprachlichen Zeichen zur Kohäsion und Kohärenz des Textes beiträgt. Es entwickelte sich eine Vorstellung von Bildern als semiotischen Objekten, in denen besondere Regeln für die Verbindung von Form und Bedeutung gelten, die sogenannte „visuelle Grammatik“ nach Kress, van Leeuwen (Kress \& van Leeuwen 1996; 2006). Ein Blick in die Fachliteratur zeigt die Begriffe „Multimodaler Text", "bimedialer", „multimedialer Text", "Sprache-Bild-Text", die markieren, dass die Kohärenz der Aussage nicht nur durch die sprachliche Komponente erzeugt wird. Die Materialität des Textes setzt sich aus verschiedenen Elementen zusammen, seine Art, Farbe, grafische Gestaltung, Layout gehören zum Interessengebiet der Sprachwissenschaft und beeinflussen die Rückschlüsse über die Prozesse des Verstehens (vgl.: Chernyavskaya, 2016; Kulikova \& Detinko, 2020; Şenöz-Ayata \& Atasoy, 2019; Schmiz, 2004; Tchernyavskaia \& Zakaryan, 2014).

In den linguistischen Studien werden mehrere Beschreibungsebenen angenommen, um die Mechanik der Verknüpfung von Sprache und Bild zu verfolgen.

Aus der Perspektive der Textstruktur kann man folgende Haupttypen von Zusammenhangen zwischen Wort und Bild unterscheiden. Erstens, Redundanz - das Bild dient als Mittel zur Doppelkodierung von Informationen, wenn das Bild das Wort unterstützt. Bei der doppelten Kodierung wird die Information durch das Bild intensiviert, emotionalisiert oder bloß „dekoriert".

Zweitens, ergibt die Kombination aus sprachlichen und visuellen Zeichen eine komplementäre Beziehung. Komplementarität liegt vor, wenn das Wort als auch das Bild sich in einer anderen Reihenfolge ergänzen können. Z.B. das Wort beginnt mit dem Thema der Nachricht, und das Bild unterstütz und entfaltet es, oder umgekehrt. Die Themaentfaltung wird sowohl durch das Wort als auch durch das Bild erzeugt. Der visuelle Teil ist ohne den verbalen in sich nicht verstehbar. Drittens, Diskrepanz oder Kontrast liegt vor bei einer ironischen Wort-Bild Verknüpfung, vgl.: (Nöth, 2000; Sandig, 2006, S. 457-460). 
Das ist an folgenden Beispielen zu verdeutlichen. Das erste Beispiel ist der Artikel „Albtraum der Partner. Ob Spanien Hilfe aus dem EU-Rettungsfonds braucht, hängt von der Sanierung der nationalen Sparkassen ab" in der Zeitung Die Zeit, 2011. Er analysiert die Krisensituation, in der sich die Staaten der Eurozone und die nationalen Banken in dieser Zeit befanden. Den Text des Artikels begleitet eine Collage: ein Bild einer 500-EuroBanknote, die aufgerissen und mit einem medizinischen Pflaster an der Rißstelle verklebt wurde. Zum Zeitpunkt der Veröffentlichung der Zeitung gab es in der europäischen Union auf allen Ebenen hitzige Diskussionen über die Zukunft der einheitlichen europäischen Währung, deren Existenz von der kritischen Finanzlage einiger Länder der Eurozone bedroht wurde. Die Semantik des schmerzhaften Zustandes der Währung wird durch das Bild des Pflasters als Attribut der medizinischen Manipulation aktualisiert. Gleichzeitig ist das Pflaster kein Heilmittel für die Krankheit und in diesem Sinne verbindet sich das Bild mit dem Diskurs der Debatte darüber, ob die Eurozone eine radikale Erneuerung und den Ausschluss mehrerer "wirtschaftlich nachzüglicher" Staaten braucht oder ob es halbe Maßnahmen ausreichen. Hier unterstützt das Bild das Thema des Textes: der Euro wird abgewertet und alle sehen die Versuche der Politiker, den Schein "zusammenzukleben“ und das Vertrauen in ihn zurückzugewinnen.

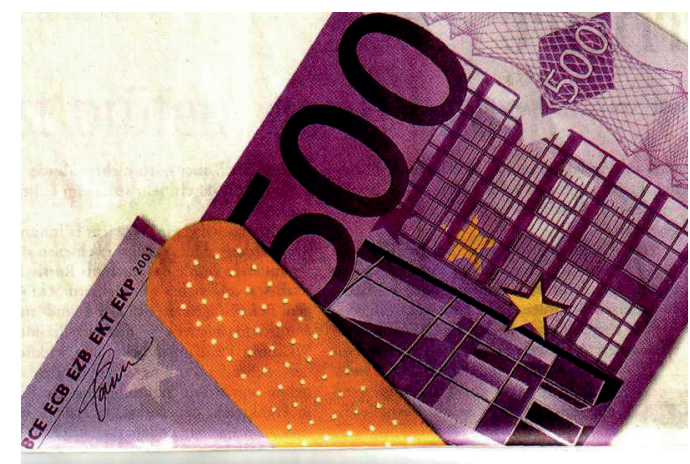

Abb.1. Albtraum der Partner. Quelle: Die Zeit, 11/2011, https://www.zeit.de/2011/11/Spanien-Sparkassen

Es kann aber auch einen semantischen Kontrast zwischen Sprache und Bild geben: So thematisiert beispielsweise der Artikel "Grün geht anders" in der Zeitung Die Zeit, 2011, das Thema Umweltschutz. Begleitet wird der Text von einer Fotocollage mit der deutschen Bundeskanzlerin Angela Merkel, deren Kostüm aus hellgrünem Gras besteht. So wird die Semantik der Spracheinheit "grün“ durch den grünen Farbcode im angenehmen Outfit der Kanzlerin verstärkt. "Grün“ verweist den Leser auf die Grünen, ein Mitglied der 
Regierungskoalition Deutschlands. Kernpunkt des Artikels ist "Schlecht gemachter Umweltschutz vergrätzt das Volk". Die Regierung verhält sich nicht grün, d.h. nicht umweltorientiert. Es sieht nur nach grün aus, was aber nicht durch echte Maßnahmen unterstützt wird. Der semantische Kontrast zwischen den positiven Konnotationen einer fröhlichen "grünen" und einer "schlecht gemachten Umweltpolitik" liegt auf der Hand. Um in der Regierungskoalition bei den Grünen zu bleiben, muss die Merkel-Regierung anders handeln und mehr Mittel für die Umwelt bereitstellen.

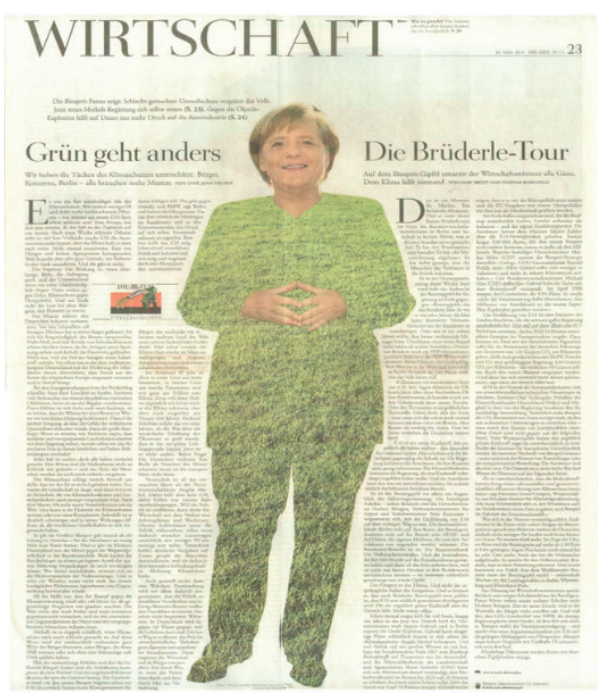

Abb. 2. Grün geht anders. Quelle: Die Zeit, 10. 03. 2011, https://www.zeit.de/2011/11/Biosprit-Umwelt-Klima

Das Visuelle wird zu einem interessanten und zunehmend gefragten Objekt der kognitiven Forschung und wird als Kognitionsinstrument erklärt. In der kognitiven Linguistik in den 2000er Jahren wird ein Ansatz zur Visualisierung aus der Perspektive einer konzeptuellen Metapher entwickelt: die Metapher hat einen verbalen und einen visuellen Modus. Die Beachtung der nonverbalen Metapher ist ein wichtiger Bestandteil in der kognitiven Forschung. Besondere Erklärungsmöglichkeiten sind auch mit visueller Metonymie verbunden. Im Gegensatz zu dem Wort und seinem symbolischen Charakter ist die visuelle Ressource ikonisch und metonymisch: das Bild spiegelt die Außenwelt als pars pro toto wider, was bedeutet, selektiv, zu einem bestimmten lokalen und zeitlichen Moment, aus einer bestimmten subjektiven Sicht. Daher ist die visuelle Semantik metonymisch im Wesentlichen (Feng, 2017, S. 445). Die Metonymie wird in der kognitiven Linguistik nicht nur und nicht so sehr als Ersatzfigur betrachtet, d.h. ein Teil stellt das 
Ganze in Bezug auf Nachbarschaft, Koexistenz und Einbeziehung in die allgemeine Situation dar, sondern als kognitive Inferenzoperation, die es ermöglicht, die Komponenten der Bedeutung zu sehen. Die Metonymie zeigt die Grenzen der Situation, in der das Verstehen geschehen soll. Sie erstellt einen situativen Rahmen mit Informationen über kausale, umständliche, assoziative Beziehungen.

Siehe folgende Beispiele: das Plakat der sozialen Werbung "Geld brennt", das in der Russischen Föderation im Rahmen des Energie - und Ressourcenschonungsprogramms veröffentlicht wurde, zeigt einen brennenden Schein in einer Glühbirne.

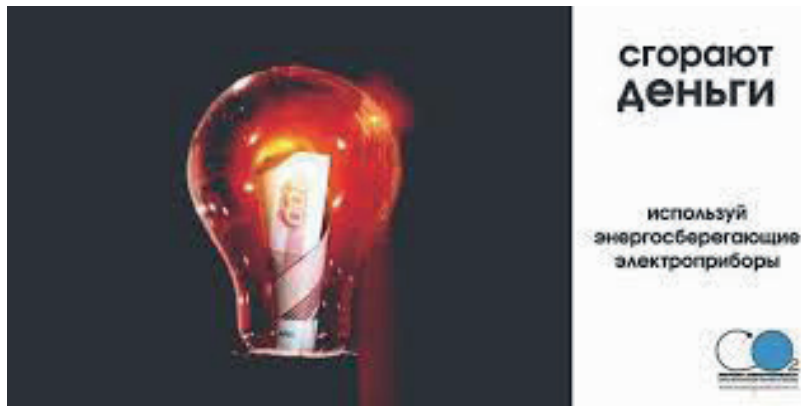

Abb. 3. Geld brennt. Quelle:

http://www.ecopolicy.ru/main.php?type_id=149\&link=change\&cnt=277

Energieeffizienz. Zentrum für Umweltpolitik Russlands

Das Plakat "Die Natur wird es nicht verdauen" ist im Rahmen des sozialen Umweltprojekts „Egal?!“ geschaffen, das 2018 in Russland ins Leben gerufen wurde. Ziel der Kampagne ist es, einen schonenden Umgang mit der Umwelt und eine umweltfreundliche Weltsicht zu fördern.

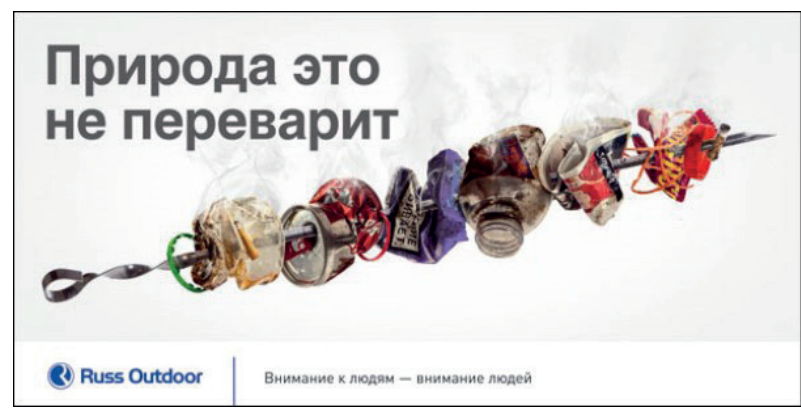

Abb. 4. Die Natur wird es nicht verdauen. Quelle: https://www.russoutdoor.ru/press/?id=1537\&sid=97 
Eine visuelle Metonymie liegt der Plakatkampagne „Kein Mensch passt in eine Schublade!“ zugrunde. Unter diesem Motto startete 2011 in der Bundesrepublik Deutschland die Antidiskriminierungsstelle des Bundes eine deutschlandweite Kampagne. Mit sechs Motiven verweist die Kampagne auf typische Kategorien („Schubladen“), in die Menschen aufgrund von Vorurteilen eingeordnet werden. Thema sind die Diskriminierungsgründe, die in Paragraf eins des Allgemeinen Gleichbehandlungsgesetzes genannt werden, nämlich Herkunft, Geschlecht, Religion, Behinderung, Alter, sexuelle Identität.

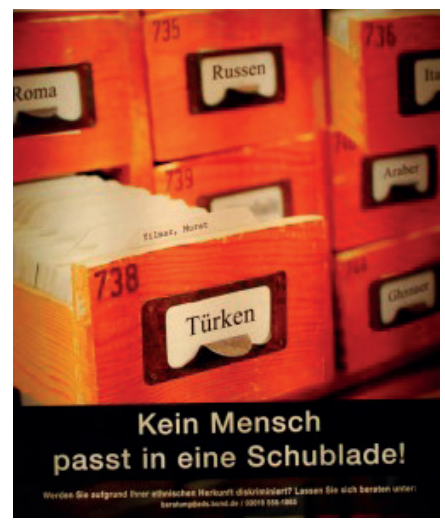

Abb.5. Kein Mensch passt in eine Schublade! Quelle:

https://www.antidiskriminierungsstelle.de/SharedDocs/Kurzmeldungen/DE/2011/nl_06_2011/ nl_06_aus_der_arbeit_der_ads_01.html?nn=8460412

\subsection{Kontext als Voraussetzung der Verstehbarkeit}

Die Forschungsbefunden zeigen das zunehmend differenzierte Verhältnis von Wort und Bild in modernen soziokulturellen Praktiken und Diskursen. Das Wahrnehmen und Verstehen eines multimodalen Textes aktiviert viele Voraussetzungen, Assoziationen und intertextuelle Verweise. Das Bild ist offen für Interpretationen. Das Verstehen des Visuellen ist kontextabhängig. Dies bedeutet, dass der Adressat die vom Bild geleitete Bedeutung auf der Grundlage seines soziokulturellen Wissens und seiner Erfahrung ableitet, wobei er sein Vorwissen und die allgemeinen Werteinstellungen berücksichtigt, die sich in der Gesellschaft entwickelt haben. Das Bild „gründet auf kontextsensible Sinnzuschreibung“ (Stöckl, 2010, S. 55).

Das folgende Beispiel stammt aus der russischen wirtschaftsanalytischen Zeitschrift Expert North West. Die Zeitschrift beleuchtet die Entwicklung von Unternehmen und die 
Wirtschaft von St. Petersburg, Nordwesten Russlands, um die Entwicklung von Privatunternehmen und positive Veränderungen in der Wirtschaft zu fördern. Von Interesse ist der analytischen Artikel „Bank Waryagi St. Petersburg“ über die Verteilung der Kräfte auf dem Petersburger Bankenmarkt zwischen lokalen Banken: großen föderalen Strukturen und Banken aus anderen Regionen, veröffentlicht 2013. Die Schlüsselthese des Artikels lautet: für einige Filialen von inländischen Banken ist es aus verschiedenen Gründen, einschließlich der Preispolitik vorteilhafter sich nicht in St. Petersburg, sondern in der Region zu registrieren und neue Kunden dort zu rekrutieren. Diese These wird durch den folgenden visuellen Text offenbart, Abb. 6. Die Bildbedeutung wird durch die Bildunterschrift expliziert: „Die Grundlage des Geschäfts der IncomingBanken sind die Firmenkunden der übergeordneten „Mutter-Kreditorganisation“.

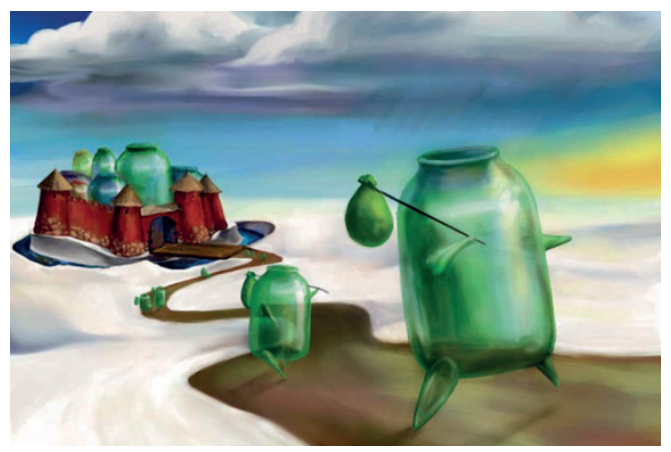

Abb. 6. Bankwaräger. Quelle:

https://expert.ru/northwest/2013/28/bankovskie-varyagi-peterburga/media/201094/

Was soll das Bild seinem Adressaten sagen? Welches Hintergrundwissen und welche postkommunikativen Effekte stehen im Mittelpunkt?

Erstens erfordert das Verständnis des visuellen Textes die Kenntnis über die Homonymie von Wörtern in der russischen Sprache: „Bank“ (deutsch: die Bank) als Finanzund Kreditinstitut und „Banka“ (deutsch: die Glasdose) als Behälter für Produkte. Dosen, hauptsächlich solche aus Glas, wie sie gezeigt werden, sind sehr charakteristisch für den vergangenen sowjetischen und modernen russischen Alltag. Sie werden zur Herstellung von hausgemachten Konserven und zur Aufbewahrung von Lebensmitteln verwendet. Auf dem Bild sehen wir das Bild mehrerer stilisierter Dosen, die von der Burg entlang einer Landstraße laufen. Dies ist offensichtlich eine visuelle Metapher für die Muttergesellschaft und ihre Tochterunternehmen in der Region. Für das Verständnis des Bildes ist die kulturspezifische Kenntnis von zentraler Bedeutung, und zwar die Kenntnis 
von den für die UdSSR typischen Praktiken der Vergangenheit. In der Sowjetunion gab es in Zeiten einer unentwickelten Marktwirtschaft und des Misstrauens gegenüber dem Bankensystem die Praxis, Bargeld nicht auf Bankeinlagen zu lagern, sondern Banknoten buchstäblich in «Banken» (Dosen) zu verstecken. Die Homonymie von „Bank-Banka“ erlangte in der sowjetischen Praxis einen charakteristischen Ruhm und wurde unter anderem in den Witzen verwendet. Die Interpretation des Bildes führt zu komplexen Assoziationsbildern der Leser, einschließlich jener, die sich auf die Stimmung der Sowjetzeit beziehen, als "Geld in Banken aufbewahrt wurde", "Drei-Liter-Banken zuverlässige Banken waren“. In diesem Fall geht es um eine Implikatur des Diskurses: nun werden zuverlässige „Banken“ selbst geschickt, um den Kunden die besten Bedingungen für die Speicherung von Ersparnissen anzubieten.

Also es entsteht ein komplexer, emotional gefärbter multimodaler Text. Seine Wirkung ist viel größer und emotionaler als die Bedeutung sprachlicher Einheiten, vgl. verbale Komponente unter dem Bild: „Die Grundlage des Geschäfts der Incoming-Banken sind die Firmenkunden der übergeordneten „Mutter-Kreditorganisation“. Sprachsemantik wirkt hier nur als eines der Elemente des komplexen Zeichens ${ }^{1}$.

Wenn visuelle Bilder in verschiedene soziokulturelle Umgebungen geraten, können sie in Bezug auf Relevanz und Bedeutung von Verbindungen, Zeichen und emotionalen Einschätzungen voneinander abweichen. Was in einer anderen Kultur hinter dem Bild steckt, ist eine wichtige Forschungsfrage, deren Beantwortung zu unseren Vorstellungen über die gegenseitige Beeinflussung des soziokulturellen Kontexts auf Zeichensysteme in der menschlichen Kommunikation beiträgt.

Wir werden unsere Erklärungen an einem Beispiel präsentieren, das auf der wissenschaftlichen Konferenz über interlinguale Kommunikation 2018 in Krasnojarsk an der Sibirischen Föderalen Universität erworben wurde. Es ist eine führende und anerkannte russische Universität, die viele internationale Projekte realisiert. Einer der Teilnehmer der Konferenz erzählte kurz von der folgenden lebhaften und sehr interessanten Situation zum weiteren Nachdenken. Unter ausländischen Studenten, die in die SFU eintraten (in diesem Fall handelte es sich anscheinend um Studenten aus

1 Dieses Beispiel wurde für die Analyse verschiedenen Adressaten in Vorträgen in Russland, Kasachstan, Weißrussland vorgeschlagen. Es ist bemerkenswert, dass moderne junge Menschen, z. B. Studierende im Fach Linguistik, diesen visuellen Text nicht verstanden, als er zur Analyse vorgeschlagen wurde. Dabei reagierten ältere Menschen, die an der bisherigen Praxis beteiligt waren, empfindlich. Dieses Bild war für sie vielsagend und transparent. 
Tunesien), wurde eine Standardumfrage durchgeführt, um die Schwierigkeiten bei der Anpassung an das neue Umfeld in Studium und Leben in Krasnojarsk zu untersuchen. Die Teilnehmer der Umfrage gaben an, dass sie alles mögen und sich bis auf einen Umstand wohl fühlen: „Sie sind es gewohnt, Milch zu trinken, und in Krasnojarsk ist es schwierig, Milch für Menschen zu finden und zu kaufen, da in Geschäften fast immer die Milch für Katzen verkauft wird." Viele Befragte antworteten auf diese Weise, und die Antwort verwirrte russische Interviewforscher. Ausländische Studierende mussten direkt zeigen, was unter "Milch für Katzen" zu verstehen ist. Und sie zeigten auf die Milchflaschen der Marke „Prostokvashino“. Auf dem Etikett von Milchprodukten ist ein Kater dargestellt, Abb. 7-8.

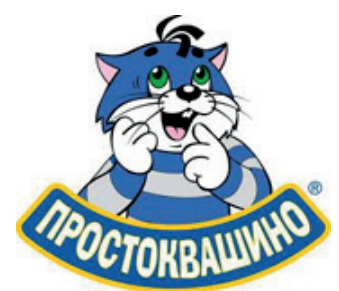

Abb.7. Marke Prostokvashino

Um auf diese Situation einzugehen, sollte man damit beginnen, dass das Thema der Analyse das sogenannte interkulturelle Missverständnis ist. Das Missverständnis ist das Ergebnis einer Diskrepanz in der Wahrnehmung des verbal-visuellen Textes im russischen Umfeld und von den Vertretern einer anderen soziokulturellen Praxis. Die intertextuellen und interdiskursiven Kompetenzen der Fremd- und Muttersprachler von Sprache und Kultur stimmten nicht überein und führten nicht zu den notwendigen und angemessenen Bezeichnungen. Das Hintergrundwissen der russischen Verbraucher umfasst Folgendes. Auf dem Etikett von Milchprodukten ist der Kater Matroskin dargestellt - ein Charakter aus der berühmten und beliebten Zeichentrickserie über einen Stadtjungen namens Onkel Fedor und seine Freunde. Onkel Fedor verlässt das Elternhaus in der Stadt und lebt im Dorf Prostokvashino zusammen mit seinem neuen Haustier, dem gestreiften Kater Matroskin, der sprechen kann. Die Helden der Geschichte wurden 1972 vom Schriftsteller E. Uspensky erfunden. Die Bilder wurden unvergesslich und traten in die sowjetische und russische Kultur ein. Der Kater Matroskin ist praktisch, sparsam und kennt sich gut mit dem köstlichen Essen aus. Der Markenname selbst, Prostokvashino", bezieht sich auf den Namen eines traditionellen russischen 
Milchprodukts, das als sehr nützlich angesehen wird². Die Marke „Prostokvashino“ nimmt den größten Marktanteil nach den Umfängen der Verkäufe auf dem Milchmarkt Russlands ein ${ }^{3}$. Heute werden die Produkte dieser Marke nicht nur in Russland, sondern auch in Weißrussland und Kasachstan verkauft.
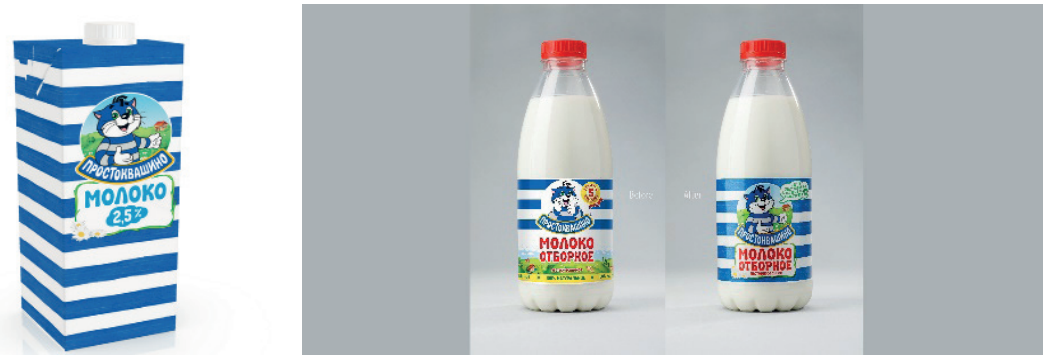

Abb.8. Prostokvashino Milchprodukte

Der visuelle Teil der Marke „Prostokvashino“ war also der Schnittpunkt vieler Assoziationen, positiver bewertender Bedeutungen und Bilder, die in der russischen Realität absolut erkennbar und „sprechend“ waren. Der Verbraucher wird vom Image eines praktischen, ökonomischen Charakters beeinflusst, der viel über hochwertigen einheimischen Produkte weiß. Wenn sich das Bild von dieser soziokulturellen Verbindung distanzierte, funktionierte er nicht als korrekter Index und übersetzte die Wahrnehmung der gesamten Situation in einen anderen Kontext. Das Bild wurde als Hinweis darauf gelesen, dass „diese Milch nicht für Menschen, sondern für Katzen ist”.

\section{Zusammenfassung}

Eine visuelle Wende, die für geistwissenschaftliche und soziale Theorien und angewandte Forschung in der Linguistik gemeinsam geworden ist, hat eine gewinnbringende Rolle des Bildes für die Bedeutungskonstruktion und -rezeption in den Mittelpunkt gerückt. Der visuelle Modus der Kommunikation wird nicht weniger bedeutend als sein der sprachliche. Wie passt das in die Sprachwissenschaft? Die Antwort auf diese Frage wird im Zusammenhang mit den folgenden Thesen gesehen.

2 Im Jahr 2002 erhielt Unimilk die Rechte für die Verwendung von Charakteren bei der Gestaltung von Produktverpackungen und in der Werbung in der Milchkategorie. In 2008 wurde das Symbol von "Prostokvashino" der charmante Kater Matroskin. Die Marketing-Entscheidung erwies sich als absolut richtig: der Umsatz stieg, der Marktanteil stieg und die Markenbekanntheit stieg ebenfalls. 
Das visuelle Bild kann und muss nicht als isolierter Teil der Kommunikation betrachtet werden. Die Autonomie des visuellen Kanals bedeutet nicht, dass es von der Sprachressource isoliert ist. Darüber hinaus kann das Bild in einer kommunikativen Situation einigermaßen unabhängig von Sprachzeichen funktionieren. Mit Bildern kann man reden, die Bilder lassen sich sehr wohl lesen. Gleichzeitig kann behauptet werden, dass ein "sprachloser Start" des kommunikativen und kognitiven Prozesses im Hintergrund des sprachlich gebildeten Vorwissens zu erklären ist.

Die Analyse der visuellen Ressource bietet einen weiteren Zugangspunkt zur Analyse der Kontextualisierung als notwendige Voraussetzung für das Verstehen. Das Bild, das zum strukturellen Element des Diskurses wird, ist offen für Interpretationen, kann für das Verständnis unbrauchbar und "hermetisch" werden, wenn es von kultur- und sozialspezifischen Praktiken distanziert wird. Die Funktion von Bildern im soziokulturellen Kontext darf nicht übersehen werden. Der visuelle Teil eines multimodalen Textes ist kein Informationsvehikal, sondern ist an der dynamischen Sinnbildung beteiligt.

Die Auseinandersetzung mit dem Visuellen bildet eine Evidenzbasis in der theoretischen Begründung, wie das gemeinsame, mit anderen Teilnehmern der kulturellen Praxis geteilte Wissen entsteht und weiter funktioniert. Die Expansion des Bildes in sozialen Praktiken erfordert von den Teilnehmern der Kommunikation reflektierte visuelle Erfahrung und eine multimodale Kompetenz. Eine solche Kompetenzen beinhaltet die Fähigkeit, ideologische, politische, kulturspezifische Implikationen von Bildern abzuleiten und zu interpretieren, die Bewertungseinstellungen zu verstehen, die hinter der visuellen Gestaltung der Bedeutungen stehen. Der Beitrag vermittelt auch Anregungen für die Analyse von multimodalen Texten in konkret umrissenen Situationen und medialen und sozialen Verwendungsbereichen.

\section{Begutachtung: Extern begutachtet.}

Interessenkonflikt: Es besteht kein Interessenkonflikt.

Finanzielle Förderung: Die Forschung wurde von der Russischen Wissenschaftsstiftung gefördert. Projekt Nummer 18-18-00442 "Mechanisms of meaning production and textualization in social narrative and performative discourses and practices", an der Im. Kant Baltischen Bundesuniversität, Kaliningrad, Russland.

\section{Peer-review: Externally peer-reviewed.}

Conflict of Interest: The authors have no conflict of interest to declare.

Grant Support: The research is supported by the Russian Science Foundation, project № 18-18-00442 "Mechanisms of meaning production and textualization in social narrative and performative discourses and practices", at the Im. Kant Baltic Federal University, Kaliningrad, Russia. 


\section{Literaturverzeichnis}

Chernyavskaya, V. (2014). Kommunikation in der Wissenschaft: Ein Plädoyer für Mehrsprachigkeit. In Alman Dili ve Edebiyatı Dergisi - Studien zur deutschen Sprache und Literatur, 2, 5-15.

Chernyavskaya, V. (2016). Multimodality versus "Logocentrism" in Persuasion. In Filologicheskie Nauki - Nauchnye Doklady Vysshei Shkoly - Philological Sciences - Scientific essays of Higher Education, 2, 3-10.

Ehlich, K. (1998). Medium Sprache. In Strohner H., Sichelschmidt L., Hielscher M. (Hrg.). Medium Sprache (S. 9-21). Frankfurt/M.: Lang.

Gumperz, J. (2002). Sharing Common Ground. In Keim I., Schütte W., (Hrsg.). Soziale Welten und kommunikative Stile (S. 47-55). Tübingen: Narr.

Feng, W. D. (2017). Metonymy and visual representation: towards a social semiotic framework of visual metonymy. In Visual Communication. 16 (4). 441-466.

Kress, G. (2010). Multimodality: A social semiotic approach to contemporary communication. Abingdon, UK: Routledge.

Kress, G. \& van Leeuwen, T. (2001). Multimodal discourse. The modes and media of contemporary communication. London: Arnold.

Kress, G. \& van Leeuwen, T. (1996 1st ed.; 2006 2nd ed.). Reading images. The grammar of visual design. London: Routledge.

Kulikova, L. V. (2019). Intercultural mediation: a discourse-analytical perspective. Voprosy Kognitivnoy Lingvistiki. № 3. 26-35. DOI: 10.20916/1812-3228-2019-3-26-35

Kulikova, L. V. \& Detinko, J. I. (2020). Discursive Construction of "Others" in the Semiotic Space of Political Communication. In E. Malyuga (Hrsg.), Functional Approach to Professional Discourse Exploration in Linguistics (S. 199-230). Singapur: Springer.

Kuße, H. \& Chernyavskaya, V. E. (2019). Culture: Towards its explanatory charge in discourse linguistics. In Vestnik Sankt-Peterburgskogo Universiteta. Yazyk i Literatura. № 3 (16). 444-462.

Kuße, H. (2012). Kulturwissenschaftliche Linguistik. Eine Einführung. Göttingen: Vandenhoeck \& Ruprecht.

Molodychenko, E. N. (2015). Axiological Dimension in the Discourse of Consumerism: The Role of Evaluative Language in the lifestyle genre. Vestnik Tomskogo gosudarstvennogo universiteta. Filologiya - Tomsk State University Journal of Philology. № 6 (38). 55-66. DOI: 10.17223/19986645/38/5.

Molodychenko, E. N. (2017). Identity and Discourse: From Social Theory to Practice of Discourse Analysis. In St. Petersburg State Polytechnic University Journal. Humanities and Social sciences. Vol. 8. №3. 122-133. DOI: 10.18721/Jhss.8312.

Nöth, W. (2000). Der Zusammenhang von Text und Bild. In Brinker, K., Antos, G., Heinemann, W. \& Sager S. (Hrsg.) Text- und Gesprächslinguistik. Ein internationales Handbuch zeitgenössischer Forschung, 1. Halbbd. (S. 489496). N.Y.: de Gruyter.

Sandig, B. (2006). Textstilistik des Deutschen. Berlin, N.Y.: de Gruyter. 
Schmitz, U. (2004). Sprache in modernen Medien. Einführung in Tatsachen und Theorien, Themen und Thesen. Berlin: Erich Schmidt.

Spitzmüller, J. (2017). ,Kultur' und das ,Kulturelle': Zur Reflexivität eines begehrten Begriffs. In Zeitschrift für Angewandte Linguistik. 67, 3-23.

Stöckl, H. (2009). The language-image-text - Theoretical and analytical inroads into semiotic complexity. In Arbeiten aus Anglistik und Amerikanistik. №34 (2). 203-226.

Stöckl, H. (2010). Sprache-Bild-Texte lesen. Bausteine zur Methodik einer Grundkompetenz. In Diekmannshenke, H., Klemm, M., Stöckl, H. (Hrg.), Bildlinguistik (S. 43-70). Berlin: Erich-Schmidt.

Şenöz-Ayata, C. \& Atasoy, İ. (2019). Ein interkultureller Blick auf Multimodalität in ausgewählten deutschen, angloamerikanischen, italienischen und türkischen Kosmetikwerbeanzeigen. Alman Dili ve Edebiyatı Dergisi - Studien zur deutschen Sprache und Literatur, 41, 1-23. https://doi.org/10.26650/sds/2019-0004

Tchernyavskaia, V. \& Zakaryan, S. (2014). Cultural marked Texts: Intercultural Dimension of Text Linguistics. In Filologicheskie Nauki - Nauchnye Doklady Vysshei Shkoly - Philological Sciences - Scientific essays of Higher Education. 2. 10-16.

Widdowson, H. G. (2004). Text, context, pretext: Critical issues in discourse analysis. (language in society). Wiley Online Library. 